frequency of a DIA4 variant — which leads to loss of protection against the toxic effects of quinones - differed between populations from China and Papua New Guinea, which were assigned to different clusters. Normally, these populations would have been grouped together as Asians, and this important difference would have been missed.

Although these results might not come as a complete surprise to those who study the complexities of population history, they do provide evidence of shortcomings in current drug trials. They also show how genetic classification might be a valuable tool for the future design of clinical trials that will have benefits both in terms of cost savings and human health.

Mark Patterson

(4) References and links ORIGINAL RESEARCH PAPER Wilson, J. F. et al. Population genetic structure or variable drug response. Nature Genet. 29, 265-269 (2001) WEB SITE

David Goldstein's lab:

http://www.ucl.ac.uk/biology/goldstein/Gold.htm
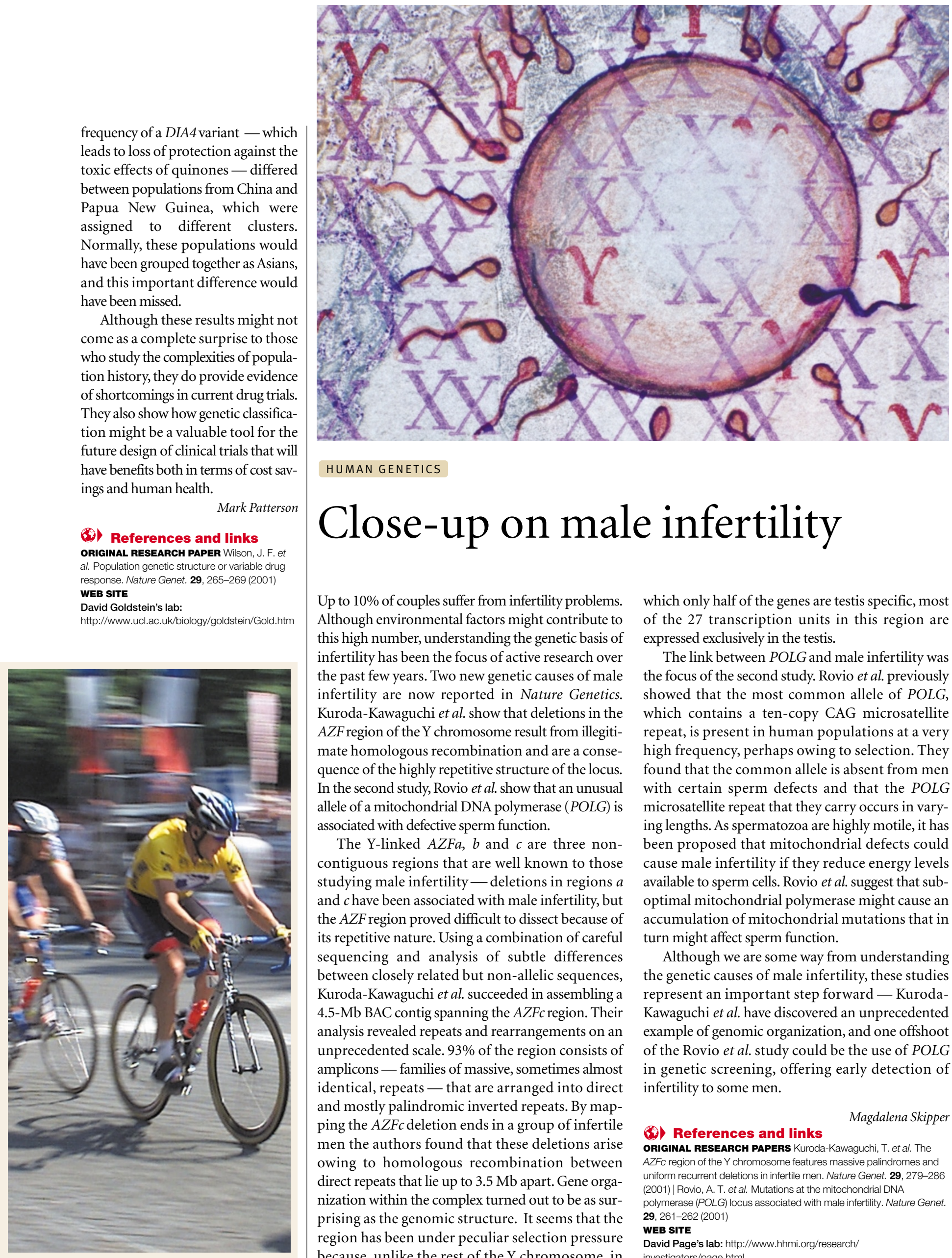

HUMAN GENETICS

\title{
Close-up on male infertility
}

Up to $10 \%$ of couples suffer from infertility problems. Although environmental factors might contribute to this high number, understanding the genetic basis of infertility has been the focus of active research over the past few years. Two new genetic causes of male infertility are now reported in Nature Genetics. Kuroda-Kawaguchi et al. show that deletions in the $A Z F$ region of the $\mathrm{Y}$ chromosome result from illegitimate homologous recombination and are a consequence of the highly repetitive structure of the locus. In the second study, Rovio et al. show that an unusual allele of a mitochondrial DNA polymerase $(P O L G)$ is associated with defective sperm function.

The Y-linked $A Z F a, b$ and $c$ are three noncontiguous regions that are well known to those studying male infertility - deletions in regions $a$ and $c$ have been associated with male infertility, but the $A Z F$ region proved difficult to dissect because of its repetitive nature. Using a combination of careful sequencing and analysis of subtle differences between closely related but non-allelic sequences, Kuroda-Kawaguchi et al. succeeded in assembling a 4.5-Mb BAC contig spanning the $A Z F c$ region. Their analysis revealed repeats and rearrangements on an unprecedented scale. $93 \%$ of the region consists of amplicons - families of massive, sometimes almost identical, repeats - that are arranged into direct and mostly palindromic inverted repeats. By mapping the $A Z F c$ deletion ends in a group of infertile men the authors found that these deletions arise owing to homologous recombination between direct repeats that lie up to $3.5 \mathrm{Mb}$ apart. Gene organization within the complex turned out to be as surprising as the genomic structure. It seems that the region has been under peculiar selection pressure because, unlike the rest of the $\mathrm{Y}$ chromosome, in which only half of the genes are testis specific, most of the 27 transcription units in this region are expressed exclusively in the testis.

The link between $P O L G$ and male infertility was the focus of the second study. Rovio et al. previously showed that the most common allele of POLG, which contains a ten-copy CAG microsatellite repeat, is present in human populations at a very high frequency, perhaps owing to selection. They found that the common allele is absent from men with certain sperm defects and that the POLG microsatellite repeat that they carry occurs in varying lengths. As spermatozoa are highly motile, it has been proposed that mitochondrial defects could cause male infertility if they reduce energy levels available to sperm cells. Rovio et al. suggest that suboptimal mitochondrial polymerase might cause an accumulation of mitochondrial mutations that in turn might affect sperm function.

Although we are some way from understanding the genetic causes of male infertility, these studies represent an important step forward - KurodaKawaguchi et al. have discovered an unprecedented example of genomic organization, and one offshoot of the Rovio et al. study could be the use of POLG in genetic screening, offering early detection of infertility to some men.

\section{(4) References and links}

Magdalena Skipper

ORIGiNal ReSearch PAPERS Kuroda-Kawaguchi, T. et al. The $A Z F C$ region of the $Y$ chromosome features massive palindromes and uniform recurrent deletions in infertile men. Nature Genet. 29, 279-286 (2001) | Rovio, A. T. et al. Mutations at the mitochondrial DNA polymerase $(P O L G)$ locus associated with male infertility. Nature Genet. 29, 261-262 (2001)

\section{WEB SITE}

David Page's lab: http://www.hhmi.org/research/

investigators/page.html 